Original Article

\title{
LOWER RIGHT QUADRANT PAIN: A SONO ANATOMICAL PERSPECTIVE
}

\author{
Vandana Tewari*, Shirin Jahan*, Rahul Ranjan** \\ *Department of Anatomy, Rama Medical College Hospital and Research Centre, Kanpur, UP. \\ ${ }^{\star \star}$ Department of Radiology, Rama Medical College Hospital and Research Centre, Kanpur, UP.
}

\begin{abstract}
Introduction: Ultrasonography has been found to be highly precise in evaluation of various abdominal masses. It is highly sensitive, non-invasive in nature and has no radiation risk.

Material \& Methods: A prospective study aiming at sonographic evaluation of 133 patients with right lower quadrant pain/mass which includes patients of all age groups and both sexes was planned. In this study, the efficiency of ultrasonography over clinical assessment in determination of the organ of origin was evaluated in a systematic manner according to anatomy of the region.
\end{abstract}

Results: The maximum number of cases belonged to gastrointestinal (GI) pathologies (54\%) followed by genitourinary (GU) pathologies (31.6\%) while 14\% non-gastrointestinal and non-genitourinary origin.

Keywords: Appendicular mass, intussusception, gastrointestinal, carcinoma caecum, genitourinary, myoma, lymph node mass.

\section{INTRODUCTION}

Ultrasound has high diagnostic accuracy in diagnosing the pathological nature of right iliac fossa masses (overall accuracy $90 \%$ ) [1] while it is $100 \%$ accurate in case of appendicular mass, in detecting normal cases, normal variants (clinically diagnosed as right iliac fossa masses), uterine mass, lymph-nodal mass and ileocolic intussusception. Differential diagnosis of lower right quadrant pain depends upon the affection of various organs present in right iliac and right lumbar region and their surrounding anatomical relations. Right lower quadrant pops up with plethora of pathological conditions which are either confined to this corner or encroach towards it and victimize a large number of patients. Differential diagnosis of lower right quadrant pain according to regional anatomy are gastrointestinal (GI) causes e.g. appendicular mass/abscess/mucocele, ileocaecal tuberculosis, intussusception, inflammatory bowel disease, carcinoma caecum. Genitourinary causes (GU) include tube ovarian masses and myomas. Non- gastrointestinal and Non-genitourinary causes include psoas abscess, ectopic kidney and post-traumatic masses. Ultrasonography (USG) has been found to be highly precise in evaluation of various abdominal masses [2] including the gastrointestinal, genitourinary and masses from various other systems. Because of its high sensitivity, non-invasive nature, lower cost, general accessibility and no radiation risk, it is widely used as a diagnostic tool. It not only provides image of mass independent of its and its vicinity organ function but also pioneers as method of choice for guided biopsies and fine needle aspirations [3]. It has been a boon to the pregnant patients by the virtue of lacking radiation risk.

\section{MATERIAL AND METHODS}

This is a prospective study of sonographic evaluation of patients with lower right quadrant pain/right iliac fossa (RIF) masses which includes patients of all age groups and both sexes. A total number of 133 patients

Address for Correspondence:

Dr. Vandana Tewari, Associate Professor, Rama Medical College Hospital and Research Centre, Rama City, GT Road, Kanpur- 209217. Mob: 9839176353 Email: vandanatewari27@yahoo.in 
referred from various clinical departments with pain/suspected RIF mass were included in the study and evaluated.

USG was done on ACCUSON 300 XE PREMIUM EDITION sonography machine with curvi-linear array transducer with frequency of $2-5 \mathrm{MHz}$, linear array transducer with frequency of $8-10 \mathrm{MHz}$ and endovaginal probe of frequency $7 \mathrm{MHz}$ in uterine and tuboovarian masses. Prior to performing USG, a verbal informed consent was obtained from patients. Detailed relevant history taking of patients was followed by thorough general, physical and abdominal examination before subjecting patients to USG examination.

\section{Technique of Examination}

Scanning was done in longitudinal and transverse directions covering all the areas of interest. For kidney and retroperitoneum, patients were also scanned in prone and lateral positions. Graded compression technique was utilized with exerting gentle compression with the high frequency transducer using both hands in same way when palpating abdomen. This technique displaced gas in bowel producing artifacts and precisely located the region of pathology by maximal tenderness if present. On gray scale sonography, following things of masses were evaluated: location, organ of origin, characteristics of mass (size, shape, margin echotexture, and calcification) and relation to adjacent organs.

This was followed by color Doppler examination with color flow mapping using low flow settings. The confirmation of data was done by fine needle aspiration cytology and biopsy, further radiological imaging (CT, barium study, intravenous urography, non-radiological tests, clinical and ultrasonographic) follow up.

\section{Statistical Analysis}

The sensitivity of detecting RIF mass by USG was $90.76 \%$ and it is $100 \%$ specific (accurate in detecting true negative). The positive predictive value of USG was $100 \%$ in detecting masses correctly i.e. $100 \%$ accurate in detecting true presence of mass. The negative predictive value was $20 \%$. Shows predictive value of negative test is $20 \%$.

\section{OBSERVATIONS AND RESULTS}

It was found that USG is $100 \%$ efficient in detecting psoas abscess, intra-abdominal abscess, parietal haematoma and lymph node mass whereas it is $75 \%$ accurate in detecting parietal abscess as compared to clinical examination which can detect psoas abscess to $75 \%$ accuracy and intra-abdominal abscess to $50 \%$ accuracy, but it can only detect one third of prietal abscess, parietal haemetoma and lymph node mass. In case of localized collection, where USG can detect one third of confirmed cases, clinical examination was unable to detect.

According to symptoms especially pain and presence/absence of mass in the affected area, clinical assessment and further sonographic evaluation was done to detect the organ of origin as per the anatomy of lower right quadrant of abdomen mainly right iliac region, partly right lumbar and right iliac region (Table 1-4, Figs. 1-8). In the present study, maximum number of cases was found in 30-45 years age group (39\%) followed by $15-30$ years age group (35.4\%), 0-15 years age group (10\%) and $45-60$ years age group (12\%). Least number of patients was seen above 60 years of age group (3\%). Male to female ratio was found to be $1: 1.5$. The common presenting symptom in all patients was pain in abdomen followed by vomiting and fever in almost $44-45 \%$, rest in line were Gl complaints followed by menstrual irregularities (Table 5). Though the mass was palpable in $68.4 \%$ cases. 
Lower right quadrant pain......

Table 1: Comparison of efficiency of USG and clinical assessment in correctly detecting the organ of origin in gastrointestinal pathologies in RIF

\begin{tabular}{|l|c|c|c|c|}
\hline \multicolumn{1}{|c|}{ Type of mass } & \multirow{2}{*}{$\begin{array}{c}\text { No. of } \\
\text { confirmed } \\
\text { cases }\end{array}$} & \multirow{2}{*}{ Percentage } & \multicolumn{2}{c|}{ No. of cases detected by organ of origin } \\
\cline { 4 - 5 } & 52 & $72.2 \%$ & By USG & By Clinical Evaluation \\
\hline Appendicular mass & 2 & $2.7 \%$ & 2 & 42 \\
\hline Carcinoma caecum & 10 & $13.8 \%$ & 9 & 0 \\
\hline Colitis & 3 & $4.16 \%$ & 3 & 8 \\
\hline Ileocolic intussusception & 3 & $4.16 \%$ & 2 & 2 \\
\hline Ileocaecal tuberculosis & 2 & $2.7 \%$ & 1 & 0 \\
\hline Carcinoma colon & 2 & $2.7 \%$ & 2 & 2 \\
\hline $\begin{array}{l}\text { Acute mesenteric } \\
\text { lymphadenitis }\end{array}$ & & & & 5 \\
\hline Total & 72 & $100 \%$ & 70 & 5 \\
\hline
\end{tabular}

Table 2: Comparison of efficiency of USG and clinical assessment in correctly detecting the organ of origin in genitourinary pathologies in RIF

\begin{tabular}{|l|c|c|c|c|}
\hline \multicolumn{1}{|c|}{ Type of mass } & \multirow{2}{*}{$\begin{array}{c}\text { No. of } \\
\text { confirmed } \\
\text { cases }\end{array}$} & \multirow{2}{*}{ Percentage } & & \multicolumn{2}{c|}{ No. of cases detected by organ of origin } \\
\cline { 4 - 5 } & 22 & $51.1 \%$ & 22 & By USG \\
\hline Right sided ovarian mass & 7 & $16.2 \%$ & 7 & 4 \\
\hline Uterine mass & 2 & $4.65 \%$ & 2 & 0 \\
\hline Renal mass & 1 & $2.32 \%$ & 1 & 0 \\
\hline Left sided ovarian mass & 5 & $11.6 \%$ & 4 & 1 \\
\hline Tuboovarian mass & 2 & $4.65 \%$ & 2 & 2 \\
\hline Right sided ectopic pregnancy & 2 & $4.65 \%$ & 2 & 0 \\
\hline Ruptured graffian follicle & 2 & $4.65 \%$ & 2 & 0 \\
\hline Ectopic iliac right kidney & 43 & $100 \%$ & 42 & 19 \\
\hline Total & & &
\end{tabular}

Table 3: Comparison of efficiency of USG and clinical examination in correctly detecting the organ of origin in non-GI and non-GU pathologies in RIF

\begin{tabular}{|l|c|c|c|c|}
\hline \multicolumn{1}{|c|}{ Type of mass } & \multirow{2}{*}{$\begin{array}{c}\text { No. of } \\
\text { confirmed } \\
\text { cases }\end{array}$} & \multirow{2}{*}{ Percentage } & & \multicolumn{2}{c|}{ No. of cases detected by organ of origin } \\
\cline { 4 - 5 } & & & By USG & By Clinical Evaluation \\
\hline Right psoas muscle abscess & 4 & $100 \%$ & 4 & 3 \\
\hline Intra-abdominal abscess & 4 & $100 \%$ & 4 & 2 \\
\hline Parietal abscess & 4 & $100 \%$ & 4 & 1 \\
\hline Parietal haematoma & 3 & $100 \%$ & 3 & 1 \\
\hline Lymph nodal mass & 2 & $100 \%$ & 2 & 1 \\
\hline Localized collection & 1 & $100 \%$ & 1 & 0 \\
\hline Total & 18 & $100 \%$ & 1 & 7 \\
\hline
\end{tabular}


J. Anat. Science, 26(1): June 2018, 38-43

Table 4: Detection of palpable RIF masses by USG

\begin{tabular}{|l|c|c|c|}
\hline & $\begin{array}{c}\text { Mass } \\
\text { Present }\end{array}$ & $\begin{array}{c}\text { Mass } \\
\text { Absent }\end{array}$ & Total \\
\hline Ultrasound +ve & 118 & 0 & 118 \\
\hline Ultrasound -ve & 12 & 3 & 15 \\
\hline Total & 130 & 3 & 133 \\
\hline
\end{tabular}

Table 5: Distribution of frequency of symptomatology in patients with RIF mass

\begin{tabular}{|l|c|c|}
\hline Symptom (n=133) & $\begin{array}{c}\text { No of } \\
\text { cases }\end{array}$ & Percentage \\
\hline Pain in abdomen & 100 & $100 \%$ \\
\hline Fever & 44 & $33.2 \%$ \\
\hline $\begin{array}{l}\text { Mass discovered by the } \\
\text { patient }\end{array}$ & 29 & $21.8 \%$ \\
\hline Vomiting & 44 & $33.0 \%$ \\
\hline $\begin{array}{l}\text { Gastrointestinal complaints } \\
\text { (diarrhea, constipation, } \\
\text { blood in stools) }\end{array}$ & 37 & $27.8 \%$ \\
\hline Menstrual irregularities & 18 & $13.5 \%$ \\
\hline Loss of appetite & 23 & $17.2 \%$ \\
\hline Weight loss & 10 & $7.5 \%$ \\
\hline Abdominal distension & 6 & $4.5 \%$ \\
\hline $\begin{array}{l}\text { Bladder complaints } \\
\text { (increase or decrease } \\
\text { frequency of micturition, } \\
\text { burning micturition, } \\
\text { hematuria) }\end{array}$ & 8 & $6.0 \%$ \\
\hline
\end{tabular}

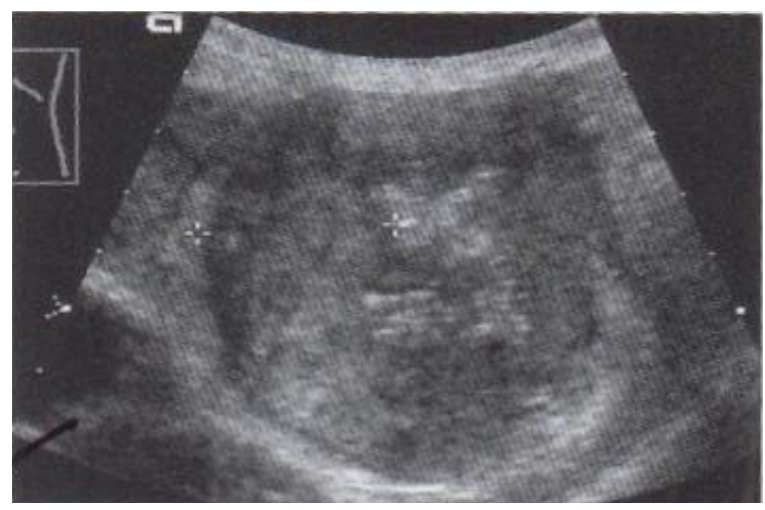

Fig. 1: USG showing Carcinoma caecum

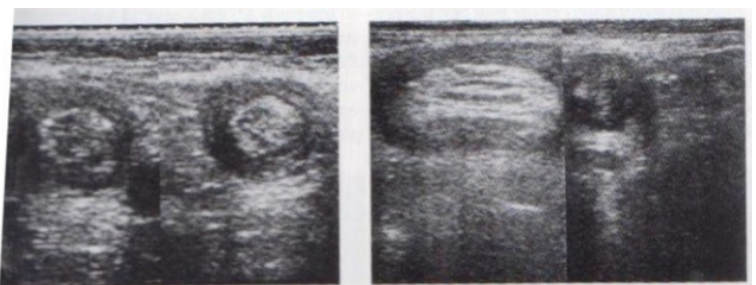

Fig. 2: USG showing intussusception \& Gut signature intussusception

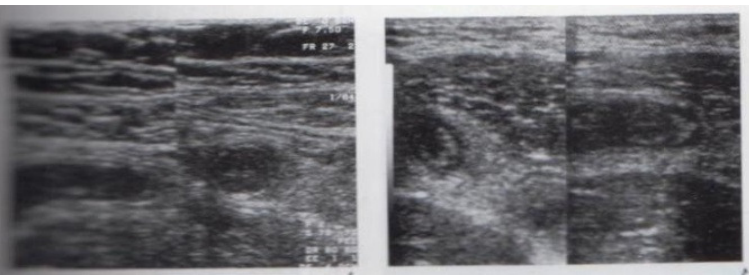

Fig. 3: USG showing appendicitis

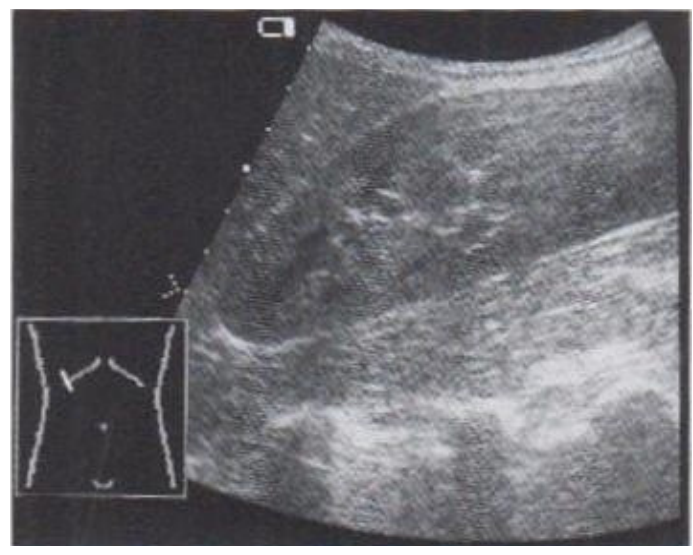

Fig. 4: USG showing caudally fused horse shoe kidney

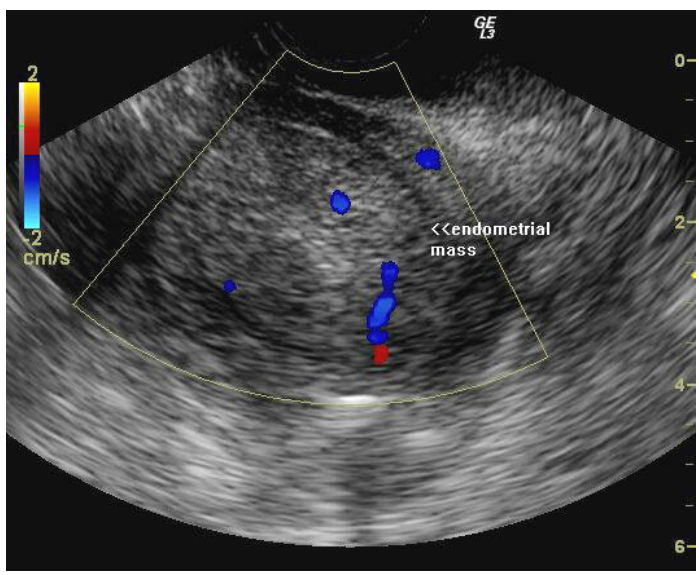

Fig. 5: USG showing Rt. lateral uterine wall myoma 


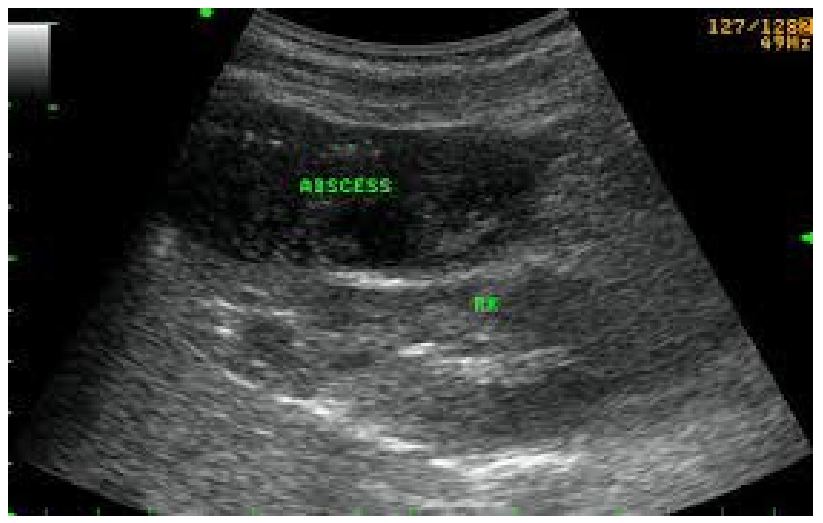

Fig. 6: USG showing Psoas abscess

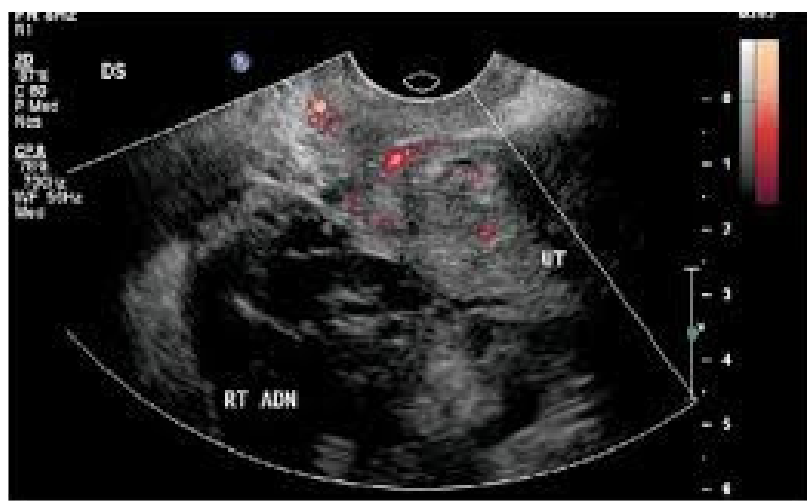

Fig. 7: USG showing heterogenous collection in pelvis secondary to ruptured ectopic gestation

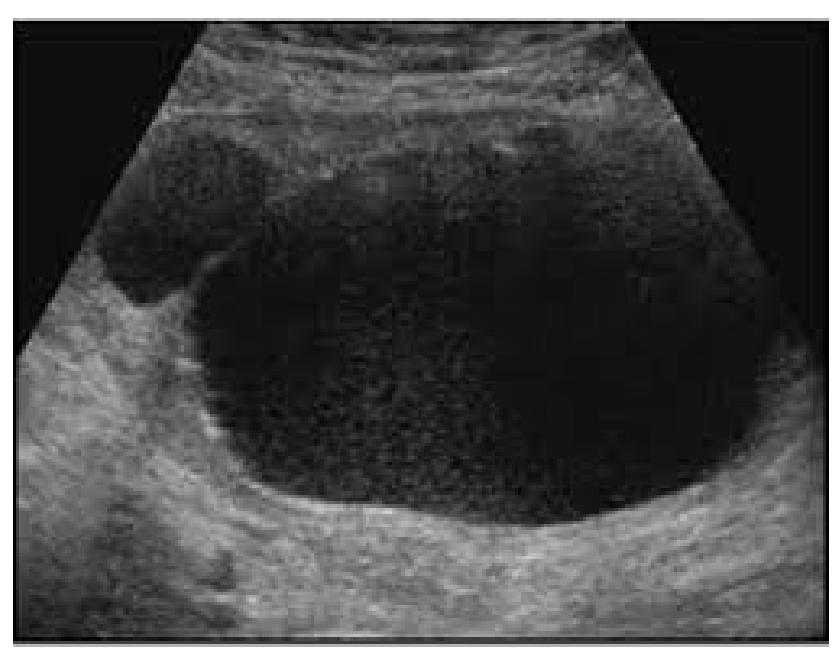

Fig. 8: USG showing Rt. ovarian echo filled cyst

\section{DISCUSSION}

The maximum number of cases belonged to gastrointestinal pathology (54\%) followed by genitourinary pathologies $(31.2 \%)$ and $14 \%$ cases belonged to non-GI non-GU origin. Majority of the lesions in the present study were found to be infective or inflammatory in origin followed by neoplastic cases, traumatic and congenital [2]. Other lesions include ileocolic intussuception [4], incisional hernia, ectopic pregnancy and normal cases.

Out of total of 72 cases of gastrointestinal pathologies, 52 cases were appendicular masses (Fig. 3) followed by 10 cases of colitis. Two cases of carcinoma colon and 2 of carcinoma caecum (Fig. 1) were found. Jadvar et al. (1997) noted that out of 10 cases of colitis, 9 were diagnosed as infectious and 1 was tubercular in nature [5]. Seven cases of infectious colitis showed positive stool culture for E. histolytica and 2 had positive blood culture for Campylobacter jejuni in a child [6], while remaining one case of tubercular colitis was finally diagnosed on colonoscopic biopsy and histopathology. In all the cases diagnosed as appendicular pathology, appendicitis was found to be most frequent finding which is similar to previous studies $[7,8]$. There were also 3 cases of ileocolic intussuception (Fig. 2) and 3 cases of ileocaecal tuberculosis. In GU pathologies out of 22 ovarian masses (Table 3), 5 were malignant, 14 benign and 3 were haemorrhagic cyst (Fig. 8). All were right sided GU pathologies except one was a huge left ovarian haemorrhagic cyst. There were 7 uterine leiomyomas extending to right iliac fossa (Fig. 5). Two cases of right adnexal ectopic pregnancy with loculated hematoma was seen extending in RIF (Fig. 7). There were 2 cases of congenital etiology which comprised of left sided caudal crossed fused renal ectopy and 1 right ectopic iliac kidney (Fig. 4). Next in number was psoas pathology which included two cases of psoas abscess (Fig. 6). USG in cases of psoas abscess revealed hypoechoic collection in right psoas muscle and bulky psoas muscle [9].

Aspiration of pus in case of abscess and haemorrhagic fluid in case of hematoma confirmed the diagnosis. The culture of the pus revealed the growth of staphylococcus. Three cases that had no masses in RIF on sonographic examination and were considered normal but clinically masses were palpated [10]. One case clinically suspected of ileocaecal tuberculosis but sonography revealed no lesion. The present study constituted appendicular masses mainly acute appendicitis [11] as maximum number of cases and 
diagnosed correctly, which shows efficiency of ultrasound in diagnosing appendicular masses especially in gravid patients with symptoms of appendicitis [12]. The second most common pathology was right ovarian mass which was almost similar to previous studies $[13,14]$ extending to RIF. Barker \& Lindsell [2] and Millard et al. [3] in their studies stated that in any patient with palpable RIF mass, USG should be the primary investigation. The initial consideration in evaluation of right lower quadrant pain or mass is whether the lesion is present or not. In the present study, 3 masses were normal variant (i.e. low lying kidney) [7]. The sensitivity and specificity of ultrasound for detection of right iliac fossa mass was found to be $100 \%$ which is similar to previous studies $[2,3]$ which also showed high sensitivity and specificity of ultrasound for detection of presence of abdominal and RIF masses respectively.

\section{CONCLUSION}

USG can detect pathologies which are inaccessible to clinical examination. It lacks hazards of radiation. It has high diagnostic accuracy in diagnosing the pathological nature of RIF masses (over all accuracy $92 \%$ ) while it is $100 \%$ accurate in appendicular mass. It is also highly accurate (100\%) in detecting normal cases and normal variants (low lying kidney) clinically diagnosed as RIF masses, uterine mass, lymph-nodal mass and ileocolic intussusception. USG had 100\% sensitivity, specificity, positive predictivity [4] and negative predictivity and accuracy in detecting presence of mass in RIF in present study.

\section{REFERENCES}

1. Aspelin P, Hildell J, Karlsson S, Sigurjonson S. Ultrasonic evaluation of palpable abdominal masses. Acta Chir Scand, 1980; 146(7):501-506.

2. Barker CS, Lindsell DRM. Ultrasound of the palpable abdominal mass. Clinical Radiology. 1990; 41:98-99.

3. Millard FC, Collins MC, Peck RJ. Ultrasound in the investigation of right iliac fossa mass. $\mathrm{Br} J$ Radiol. 1991; 64: 17-19.

4. Verschelden P, Filiatrault D, Garel L, Grignon A, Perreault G, Boisvert $J$ et al. Intussuscetion in children: Reliability of US in diagnosis-A Prospective Study. Radiology. 1992; 184:741-744.

5. Jadvar H, Mindeizun RE, Olcott EW, Levitt DB. Still a great mimicker: Abdominal Tuberculosis. AJR, 1997; 168:1455-1460.

6. Puylaert JB, Vander Zant FM, Mutsaers JA. Infectious ileocecitis caused by Yersinia, Campylobacter, and Salmonella; clinical, radiological and US findings. Eur Radiol. 1997;7(1):3-9.
7. Das S. A Manual on Clinical Surgery. 5th edition, S. Das Publishers, Calcutta, 2000; 392-394.

8. Andersson RE. Meta-analysis of the clinical and laboratory diagnosis of appendicitis. Br J Surg. 2004; 91:28-37.

9. Yadav RP, Agrawal CS, Adhikary S, Kumar M, Regmi R, Amatya R, Gupta RK. Iliopsoas abscess: Analysis and perspectives from an endemic region of Eastern Nepal. Kathmandu University Medical Journal. 2007; 5(20):497-500.

10. Standring S. Gray"s Anatomy: The Anatomical Basis of Clinical Practice. $40^{\text {th }}$ Ed., Churchill Livingstone Elseviers Publishers, London, 2008; 1125-1162.

11. Toorenvliet BR, Wiersma F, Bakker RF. Routine ultrasound and limited computed tomog raphy for the diagnosis of acute appendicitis. World J Surg. 2010; 34(10):2278-85.

12. Lehnert BE, Gross JA, Linnau KF et al. Utility of ultrasound for evaluating the appendix during the second and third trimester of pregnancy. Emerg Radiol. 2012; 19(4):293-9.

13. Malhotra A, Tarafdar S, Tayade A. Benign versus malignant adnexal masses: Does addition of Color and Spectral Doppler over and above the Gray Scale Ultrasound improves efficacy? Sch J App Med Sci. 2016; 4(1A):62-74.

14. Tarafdar S, Malhotra A, Tayade A. Acute Abdomen: Role of Ultrasonography in differentiation of common clinical mimics of appendicitis. GJRA. 2016; 5(4):20-23. 